\title{
Tailored $\mathrm{CeO}_{2}$ Nanoparticles Surface in Free Radical Bulk Polymerization of Methyl Methacrylate
}

\author{
Özge Tunusoğlu ${ }^{\dagger}$ and Mustafa M. Demir*, ${ }^{\dagger, \ddagger}$
}

${ }^{\dagger}$ Department of Chemistry and Department of Material Science and Engineering, Izmir Institute of Technology 35430 Gülbahce, Urla, Izmir, Turkey

\section{Supporting Information}

ABSTRACT: Polymerization of monomer/nanoparticle dispersion, namely in situ polymerization, has been frequently used for the fabrication of polymer nanocomposites. However, the interference of nanoparticle surface with polymerization in the course of composite formation has been tacitly neglected. In this work, surface-functionalized ceria nanoparticles were prepared using various capping agents: 3-(mercaptopropyl) trimethoxy silane, thioglycolic acid, 3-mercaptopropionic acid, and hexadecyltrimethyl ammonium bromide. Both in situ and ex situ approaches were applied for surface functionalization. The particles were dispersed into methyl methacrylate and free radical polymerization was carried out. The process of nanocomposite formation was examined in terms of conversion, molecular weight, and molecular weight distribution. The polymerization responded merely to the in situ functionalized particles. Regardless of the capping agents used, the particles function as a retarder and inhibitor. Their interaction with polymerization medium showed many complexities such that molecular weight was found to be strongly dependent on the capping agent employed.

\section{INTRODUCTION}

Composites of polymer matrices and nanoscale inorganic building blocks have shown the development of new or at least improved material properties such as high/low refractive index, ${ }^{1}$ thermal stability, ${ }^{2}$ toughness, ${ }^{3}$ high yield strength, ${ }^{4}$ high conductivity, ${ }^{5}$ gas and solvent transport, ${ }^{6,7}$ wear and scratch resistance, ${ }^{8-10}$ etc. Interfacial attraction has been recognized as an important parameter for composite materials. It determined the processability, microstructure, and accordingly the macroscopic properties of the resulting material. ${ }^{11}$ This is because fundamental understanding of polymerinorganic interface is a critical challenge in polymer nanocomposites.

In situ polymerization has attracted considerable attention for the preparation of homogeneous nanocomposites. ${ }^{11}$ It involves dispersion of particles into a monomer or monomer formulation, and then subsequent polymerization is carried out in the presence of the nanoparticles. Mixing polymer chains and nanosized particles at the molecular level, which is in fact the ultimate goal for polymer nanocomposites, can be readily achieved when this process is employed. In conjunction with this advantage, it has been shown that the composites prepared by in situ polymerization offer materials with better physical properties compared to the composite prepared by simple blending, although identical material components are used at identical composition. Recent examples could be PMMA/ZnO for thermal stability, ${ }^{12,13}$ polyimide/multi walled carbon nanotubes for high conductivity, ${ }^{14}$ poly $(p$-phenylene benzobisoxazole)/multi walled carbon nanotubes for UV-vis absorption, ${ }^{15}$ and polypropylene/ clay $^{16}$ for better mechanical properties. The reason for the improvements in material properties has been usually attributed to homogeneous internal structure. Besides, we think that the improvements may also be a consequence of interfacial attraction between particle surface and propagating polymer chain. Considering that polymer chains are initiated and grow in the presence of nanoparticles, an interaction with the particle surface and reactive propagating chain is highly possible. For instance, our group previously proposed that hydroxyl groups available on the $\mathrm{ZnO}$ surface may induce a degenerative transfer in free radical polymerization of MMA initiated by $2,2^{\prime}$-azobis(isobutyronitrile) $(\text { AIBN })^{2,17}$ We claimed that this interaction suppresses the formation of chains having vinylidene end groups and head-tohead links. Thermal stability of the composite material, as a result, is remarkably improved compared to the same composites prepared by blending. The interaction of propagating radicals with surface hydroxyl groups suppresses the undesirable gel effect, which leads to a better control over both the heat evolution during the late stages of polymerization and the molecular weight distribution. The improvement in both thermal properties and the polymerization process of $\mathrm{PMMA} / \mathrm{ZnO}$ was seen as a consequence of the interaction of surface hydroxyl groups and growing polymer chains. According to the best of our knowledge, there is no clear picture about the effect of other functional groups such as $-\mathrm{COOH},-\mathrm{SH},-\mathrm{OCH}_{3}$, and aliphatic chains on the in situ polymerization process. Therefore, it is important to understand the effect of nanoparticles functionalized with these chemical groups on the process of in situ polymerization. In this work, organophilic ceria nanoparticles were prepared using various capping agents 3-(mercaptopropyl) trimethoxy silane (MPS), hexadecyltrimethyl ammonium bromide (CTAB), 3mercapto propionic acid (3-MPA), and thioglycolic acid

Received: June 13, 2013

Revised: August 13, 2013

Accepted: August 16, 2013

Published: August 16, 2013 
Scheme 1. Synthesis of $\mathrm{CeO}_{2}$ Nanoparticles Showing In Situ and Ex Situ Surface Functionalization Processes Step-by-Step in-situ surface modification:

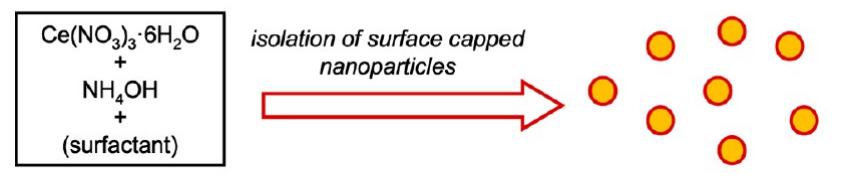

ex-situ surface modification:

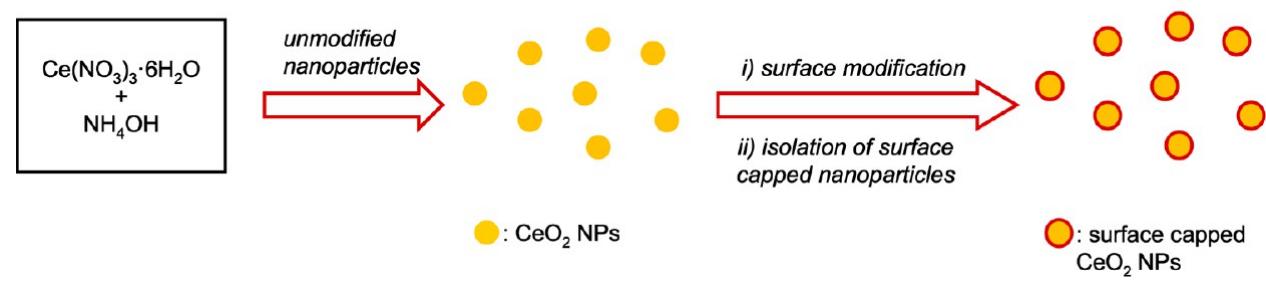

(TGA) such that the surface of particles is varied with different functional groups. Both in situ and ex situ approaches were employed for surface functionalization. The surface-functionalized particles either in situ or ex situ are referenced as ceria(-capping agent), e.g., ceria(-CTAB), throughout the text. The particles were dispersed into bulk MMA and the effect of particle surface on the process of in situ bulk polymerization was studied. MMA is a frequently used monomer in industry for the production of commercial organic glass. ${ }^{18}$ It has been commonly polymerized by the free radical mechanism, which is well studied in the literature. ${ }^{19}$ Furthermore, ceria is a substrate on which various functional groups can be chemically grafted. ${ }^{20}$ It has unique physical properties such as high refractive index, ${ }^{1}$ absorbance over UVA region, ${ }^{21}$ and high catalytic activity for water gas shift reaction, ${ }^{22}$ fuel cells, ${ }^{23,24}$ and oxidation. ${ }^{25}$ The combination of MMA and ceria can be a good model system to study the reactivity of particle surface on free radical polymerization in nanocomposite formation. Bulk polymerization was carried out since it allows rapid process with high conversion, high purity, and ease of handling. ${ }^{26}$ Conversion and molecular weight (MW) were examined by gravimetry and gel permeation chromatography (GPC), respectively. Thermal decomposition was determined by thermogravimetric analysis (TGA) and glass transition temperature $\left(T_{\mathrm{g}}\right)$ of the composites was measured by differential scanning calorimeter (DSC). Through these approaches we attempt to identify the interactions of surface functionality with propagating PMMA radicals. Polymerizations of neat MMA, MMA in the presence of mere capping agents, and MMA in the presence of unfunctionalized ceria nanoparticles were studied as control systems. The overall results suggest that polymerization is retarded in the presence of the in situ functionalized particles, independent of the chemistry of the capping agents. Moreover, MW and MWD were found to strongly depend on the chemistry of capping agents.

\section{EXPERIMENTAL SECTION}

Materials. $\mathrm{Ce}\left(\mathrm{NO}_{3}\right)_{3} \cdot 6 \mathrm{H}_{2} \mathrm{O}$ (Sigma-Aldrich) and $\mathrm{NH}_{4} \mathrm{OH}$ (Merck) were used as precursor agents. 3-Mercaptopropyl trimethoxy silane (MPS, Aldrich, 95\%), hexadecyltrimethyl ammonium bromide ( $\mathrm{CTAB}$, Aldrich), 3-mercapto propionic acid (3-MPA, Alfa Aesar), and thioglycolic acid (TGA, Merck) were used as capping agents. Methyl methacrylate (MMA, 99\%
(GC)) and benzoyl peroxide (BPO, Fluka, purum $\geq 97.0 \% \mathrm{RT}$ ) were used as monomer and initiator, respectively.

Preparation of Ceria Nanoparticles. The preparation of in situ surface functionalized $\mathrm{CeO}_{2}$ nanoparticles has been described elsewhere. ${ }^{27}$ Briefly, an aqueous solution of $0.05 \mathrm{M}$ of $\mathrm{Ce}\left(\mathrm{NO}_{3}\right)_{3} \cdot 6 \mathrm{H}_{2} \mathrm{O}$ and $0.5 \mathrm{M}$ of $\mathrm{NH}_{4} \mathrm{OH}$ were mixed. Capping agent $\left(2.65 \times 10^{-5} \mathrm{~mol}\right)$ was introduced into the system right after the achievement of homogeneous solution. The solution was stirred for $3 \mathrm{~h}$ for surface functionalization. The nanoparticles were isolated from the solution mixture by centrifugation at $6,000 \mathrm{rpm}$ for $1 \mathrm{~h}$. The particles were dried in a vacuum oven. For ex situ functionalized $\mathrm{CeO}_{2}$ particles, a two step process was applied. First, unfunctionalized particles were obtained by precipitation of $\mathrm{Ce}\left(\mathrm{NO}_{3}\right)_{3} \cdot 6 \mathrm{H}_{2} \mathrm{O}$ and $\mathrm{NH}_{4} \mathrm{OH}$ and the particles were dispersed into $50 \mathrm{~mL}$ of ethanol. This alcoholic dispersion was sonicated in ultrasonic bath in order to achieve better dispersion. Second, the desired capping agent was added into the dispersion and the mixture was allowed for surface functionalization for $12 \mathrm{~h}$. The particles were obtained by centrifugation and dried under vacuum.

Preparation of PMMA/Ceria Nanoparticle Nanocomposites. A sample of $30 \mathrm{mg}$ of ceria powder was dispersed into $1 \mathrm{~mL}$ of MMA. The dispersion was sonicated for $30 \mathrm{~min}$. In order to achieve complete wetting of the particle surface, the dispersions were kept overnight at room temperature. BPO (1.5 wt \%) was added to the dispersion after a second ultrasonication for $30 \mathrm{~min}$. Prior to polymerization, three cycles of a freeze-thaw processes were applied. Polymerization was performed at $60{ }^{\circ} \mathrm{C}$ and stopped after desired reaction time $(15,30,60,120,180,240,300$, and $1020 \mathrm{~min})$ by quenching to room temperature.

Characterization. The crystal structure of synthesized particles were characterized by Philips X'pert Pro X-ray Diffractrometer with a $\mathrm{Cu} \mathrm{K} \alpha$ radiation source $(\lambda=1.54 \AA)$. The average size of $\mathrm{CeO}_{2}$ crystallites was determined by Scherrer's equation

$$
t=\frac{0.9 \lambda}{B \cos \theta}
$$

where $t$ is the diameter of the particle, $\lambda$ is the wavelength of $\mathrm{Cu}$ $\mathrm{K} \alpha$ radiation, and $B$ is the line broadening. Transmission electron microscopy (TEM) was performed using Zeiss EM 902 (Goettingen, Germany) microscope operated at $80 \mathrm{kV}$. Fourier transform infrared (FTIR) spectra were registered by 
Table 1. Characteristic Properties of Synthesized the Ceria Nanoparticles and Particle Domains

\begin{tabular}{|c|c|c|c|c|c|c|}
\hline & \multicolumn{3}{|c|}{ in situ } & \multicolumn{3}{|c|}{ ex situ } \\
\hline & $\begin{array}{l}\text { crystal size }^{a} \\
(\mathrm{~nm})\end{array}$ & $\begin{array}{c}\text { hydrodynamic radius }^{b} \\
(\mathrm{~nm})\end{array}$ & $\begin{array}{c}\text { graft density }^{c} \\
\left(\text { molecules } / \mathrm{nm}^{2}\right)\end{array}$ & $\begin{array}{l}\text { crystal size }{ }^{a} \\
(\mathrm{~nm})\end{array}$ & $\begin{array}{c}\text { hydrodynamic }_{(\mathrm{nm})} \text { radius }^{b} \\
\end{array}$ & $\begin{array}{c}\text { graft density }^{c} \\
\left(\text { molecules } / \mathrm{nm}^{2}\right)\end{array}$ \\
\hline unmodified ceria & 8 & 600 & no grafting & 11 & 110 & no grafting \\
\hline ceria(-MPS) & 4 & 40 & 2.5 & 11 & 260 & negligible grafting \\
\hline ceria(-CTAB) & 10 & 20 & 0.9 & 9 & 90 & 0.1 \\
\hline ceria(-MPA) & 6 & 600 & 2.4 & 11 & 120 & 0.8 \\
\hline ceria(-TGA) & 6 & 600 & 4.1 & 10 & 100 & 3.9 \\
\hline
\end{tabular}

${ }^{a}$ Crystal size from XRD estimated by Scherrer's equation. ${ }^{b}$ Mean diameter of number distribution obtained from DLS in MMA. ${ }^{c}$ Graft density estimated by mass loss and size of the particles.

Scheme 2. Molecular Structures of the Capping Agents<smiles>CO[Si](C)(CCCS)OC</smiles>

3-mercaptopropyl trimethoxy silane (MPS)<smiles>O=C(O)CCS</smiles>

3-mercaptopropionic acid (MPA)

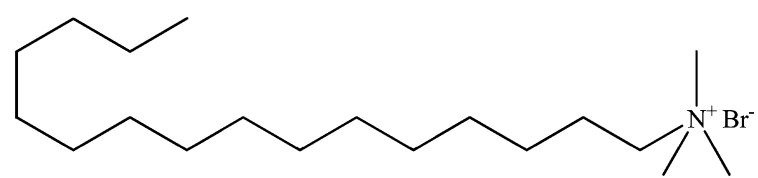

Hexadecyltrimethyl ammonium bromide (CTAB)<smiles>O=C(O)CS</smiles>

Thioglycolic acid (TGA) using a Perkin-Elmer Spectrum 100 instrument (Perkin-Elmer, Massachusetts) within $4000-400 \mathrm{~cm}^{-1}$ wavenumber, 20 scans, and resolution of $4 \mathrm{~cm}^{-1}$. The size distributions of particles dispersed in MMA were investigated by dynamic light scattering (DLS) Malvern Zetasizer Nano-ZS Nano Series (Malvern, Worcestershire). The viscosity and refractive index of the medium were specified as $0.584 \mathrm{mPa} \cdot \mathrm{s}$ and $1.414\left(20^{\circ} \mathrm{C}\right)$, respectively. Differential scanning calorimetry (DSC) was performed by using TA Instrument Q10 (New Castle), operating between 25 and $250{ }^{\circ} \mathrm{C}$ by an increment of $10{ }^{\circ} \mathrm{C}$. $\mathrm{min}^{-1}$. Thermogravimetric analysis was utilized in a PerkinElmer Diamond TG/DTA thermobalance (Perkin-Elmer, Massachusetts) by heating from room temperature to 1000 ${ }^{\circ} \mathrm{C}$ at a heating rate of $10{ }^{\circ} \mathrm{C} \cdot \mathrm{min}^{-1}$. Conversion of MMA to PMMA was determined gravimetrically. Initial weight of polymerization reactor including test tube, monomer, initiator, and ceria nanoparticles is subtracted from the final weight of the reaction system after complete removal of residual monomer under vacuum. AFM measurements were performed by Nanoscope IV (Digital Instruments-MMSPM, New York) instrument in tapping mode scanning $5 \times 5,3 \times 3$, and $1 \times 1$ $\mu \mathrm{m}^{2}$ area, respectively.

\section{RESULTS}

3.1. Preparation of Surface Functionalized $\mathrm{CeO}_{2}$ Particles. The in situ polymerization process is based on the dispersion of nanoparticles in monomer or monomer solution. When inorganic particles are dispersed into a liquid medium, they gradually sediment due to their higher density compared to that of the organic medium. Sedimentation disturbs the stability of the dispersion and the concentration of particles will no longer be uniform throughout the dispersion volume. Surface functionalization has been usually applied to prevent sedimentation. There are two main approaches for surface functionalization of nanoparticles: (i) in situ and (ii) ex situ. Scheme 1 shows a cartoon demonstration of both approaches we employed in this study. In former, the precursors $\left[\mathrm{Ce}\left(\mathrm{NO}_{3}\right)_{3} \cdot 6 \mathrm{H}_{2} \mathrm{O}\right.$ and $\left.\mathrm{NH}_{4} \mathrm{OH}\right]$ and a capping agent are added to the reaction medium at the beginning of the reaction. Nanosized ceria particles are nucleated, grown, and surfacefunctionalized in the same medium at one single step. On the other hand, the latter approach takes two steps. First, neat ceria particles are synthesized and these particles are isolated from the reaction mixture. Second, they are redispersed in ethanol, and allowed for surface functionalization. The main difference of both approaches lies in timing of capping agent addition into the reaction medium. This difference in the procedure causes severe changes both in morphology and in physical properties of the resulting materials. Table 1 presents the results of characterization of the particle obtained by both approaches. Four different capping agents were employed in this study for surface functionalization. Their structures are presented in Scheme 2. X-ray diffraction (XRD) pattern of the functionalized $\mathrm{CeO}_{2}$ nanoparticles is shown in Figure $\mathrm{S} 1$. The reflections in both diffractograms were perfectly indexed with cubic ceria structure (JCPDS Card No. 81-0792). Crystal size was determined by using Scherrer's equation, and the results are given in the first and the fourth column of Table 1 . The size of in situ functionalized particles varies between 4 and $10 \mathrm{~nm}$; on the other hand, the one of ex situ functionalized particles lies in a narrow range of 9-11 $\mathrm{nm}$. Capping agents in the process of in situ functionalization chemically grafts to the surface at early stages of particle formation and suppressed the undesirable particle growth, therefore smaller crystals are observed. The relative height of the XRD reflections coming from (111) and (200) planes hints about the geometrical shape 
of crystallite of the particles. ${ }^{28}$ It is usually assumed that at early stages of particle formation, ceria particles have octahedral morphology having (111) and (200) planes. ${ }^{29}$ The addition of capping agents interferes with the development of ceria nanocrystals. In the process of in situ functionalization, capping agents may selectively adsorb to the (200) plane so that growth of the crystal through the (200) plane is suppressed. Then, the (111) plane grows relatively faster, and eventually, a cubic structure is formed. In the process of ex situ functionalization, on the other hand, the particles have cubohedral structure as in the case of unfunctional particles. Since the particles are already matured when the capping is added, the addition of capping agents has almost no influence on the final morphology of the ceria particles, at least in the range of the conditions we employed. Figure 1 presents TEM micrographs of in situ and ex (a)

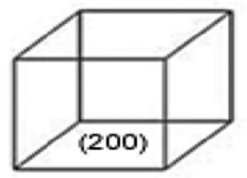

cubic
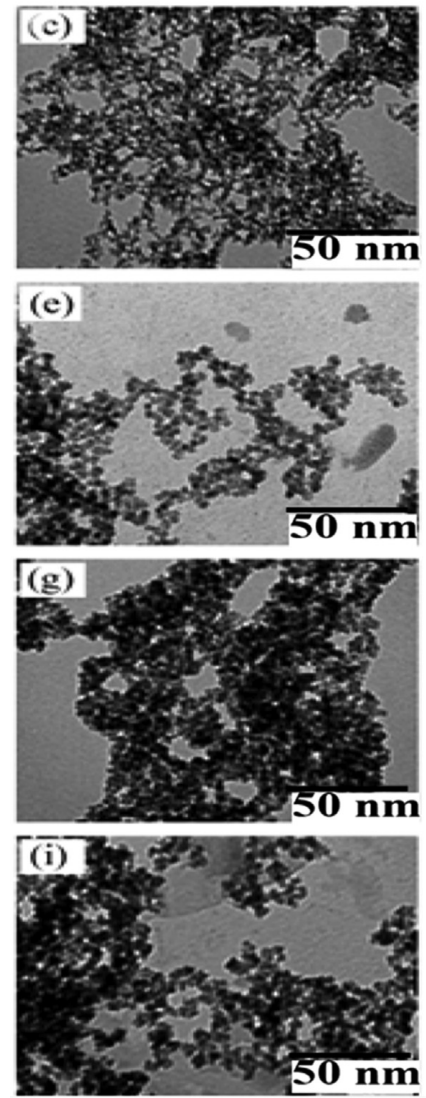

(b)

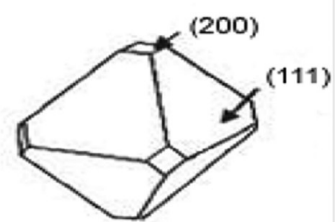

truncated octahedral
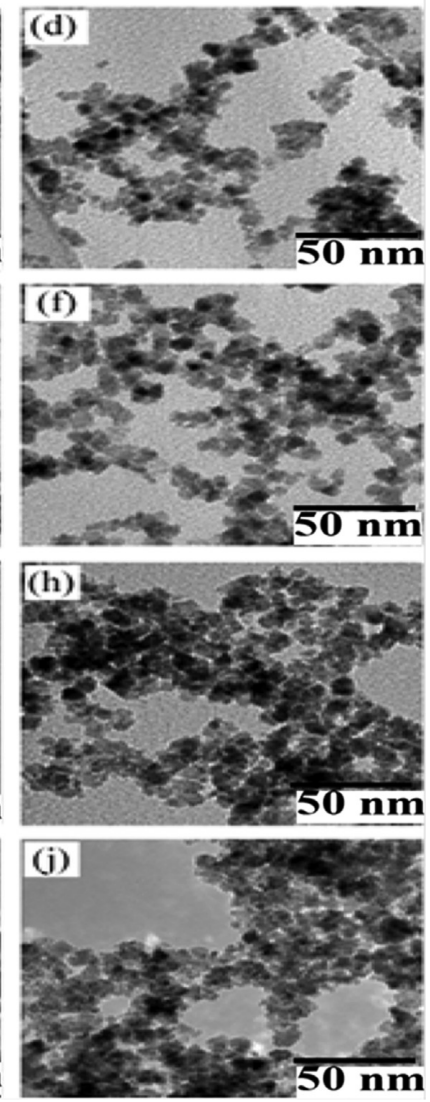

Figure 1. Structural models of $(\mathrm{a}, \mathrm{b})$ in situ and ex situ functionalized particles and TEM micrographs of in situ and ex situ (c, d) MPS, (e, f) CTAB, (g, h) MPA, and (i, j) TGA functionalized $\mathrm{CeO}_{2}$ nanoparticles.

situ functionalized ceria nanoparticles. The former ones are smaller and more uniform in terms of size. The size observed on TEM images is in the same line with the result of XRD.

Surface chemistry of the in situ functionalized particles was studied by vibrational spectroscopy. FTIR provides information about the functional groups present on the particle surface. The spectra of both in situ and ex situ functionalized particles were compared with the spectrum of neat capping agents. The wavenumbers of vibrational bands are given in Figure S2. The appearance/disappearance or the shifting/broadening in the characteristic vibrations of pure surfactant molecules may provide information about the interaction of capping agents with the particle surface. ${ }^{27}$ The unfunctionalized particles have $-\mathrm{NH}_{2}$ and $-\mathrm{CH}_{2}$ stretching most probably due to the presence of residual $\mathrm{NH}_{4} \mathrm{OH}$ and ethanol molecules coming from the preparation steps. All of the capping agents (except CTAB) have $-\mathrm{SH}$ groups. TGA and MPA are structurally similar capping agents. They have $-\mathrm{COOH}$ end groups, which appear at around $1550 \mathrm{~cm}^{-1}$. The capping agent grafts to the surface via $-\mathrm{COOH}$ groups, and $-\mathrm{SH}$ becomes available on the particle surface. The vibration of $\mathrm{SH}$ is generally observed around $2800-2500 \mathrm{~cm}^{-1}$. This band is not resolved in our spectra of thiol containing capping agents due to the low molar absorption coefficient on the particle surface. ${ }^{23}$ In the spectrum of MPS functionalized particles, a new band approximately at $1100 \mathrm{~cm}^{-1}$ appears, which can be attributed to the $\mathrm{Si}-\mathrm{O}-\mathrm{C}$ bond. The formation of this band can suggest that MPS chemically grafts to the particle surface. In the spectrum of CTAB-capped particles, the vibration of $-\mathrm{CH}_{2}$ bending at 1346 $\mathrm{cm}^{-1}$ is remarkably enhanced. Moreover, a Coulombic interaction can be formed between quaternary ammonium salt and ceria surface. The overall results based on vibrational spectroscopy indicate that the particle surface is indeed functionalized with these capping agents. Note that vibrational spectroscopy is a qualitative technique that shows the presence of the capping agents on the particle surface; however, it does not give information about the mechanism of interaction and quantitative information about the density of the capping agents on particles surface.

The level of particle dispersion in MMA was studied by DLS. Mean diameter of neat, in situ functionalized, and ex situ functionalized ceria particles are shown in the third and sixth column of Table 1. The unfunctionalized particles exhibited large particle domains. The mean diameter is nearly $0.1 \mu \mathrm{m}$ and the tail of distribution extends to several micrometers. Hydroxyl groups inevitably present at the surface of the particles may cause severe particle aggregation due to strong hydrogen bonding interactions between the particles coupled with poor compatibility of the particles with the surrounding monomer. Functional groups on the particle surface may strenghten this interaction and results in better dispersibility of the particles. For instance, the diameter of particles functionalized with MPS and $\mathrm{CTAB}$ in situ have average size of 40 and $20 \mathrm{~nm}$, respectively. These capping agents make the particle surface hydrophobic enough to disperse the particles as small domains. In case of MPA and TGA functionalized particles, the domain size is on the order of half $\mu \mathrm{m}$. This size is very large, i.e. surface functionalization makes the dispersibility worse than the unfunctionalized particles. It can be claimed that capping agents are hydrophilic so the capped particles accordingly have hydrophilic nature. This is because the particles undergo aggregation and show large domain size. On the other hand, the domain size of ex situ modified particles, regardless of the capping agents employed, was found to be in the range of $0.10-0.25 \mu \mathrm{m}$. Thus, they are not sufficiently functionalized with organophilic capping agents.

Sedimentation of the particles is related to dispersibility of the particles, which strongly depends on both particle size and enthalpic interaction of particle surface with surrounding 
medium. Smaller particle size and high interfacial attraction provide colloidal stability that remarkably retards the undesirable sedimentation. Among the all particles employed in this research, ceria(-CTAB) functionalized in situ, the smallest particles, provided the most stable colloidal stability in MMA.

Thermogravimetric analysis under nitrogen flow was studied for thermal degradation of both in situ and ex situ functionalized cerium oxide nanoparticles. Figure 2 shows a

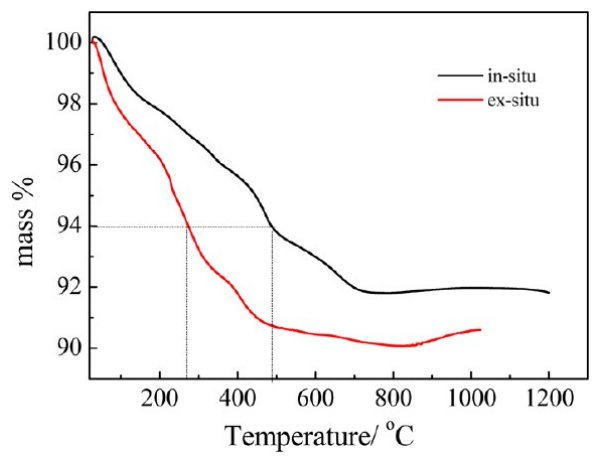

Figure 2. Thermogram of CTAB-capped ceria particles prepared by in situ and ex situ.

representative thermogram showing the mass loss of CTABfunctionalized $\mathrm{CeO}_{2}$ nanoparticles prepared by both in situ and ex situ approaches. While 6\% mass loss in TGA of in situ functionalized ceria (-CTAB) particles occur at $500{ }^{\circ} \mathrm{C}$, the same amount of mass loss is seen at $300{ }^{\circ} \mathrm{C}$ when particles are functionalized ex situ. Thus, in situ functionalized particles turn out to be thermally more stable than the one prepared by ex situ functionalization. The capping agents are more tightly adsorbed to the particle surface in the process of in situ functionalization opposing to the ones used in ex situ functionalized particles. By knowing the mass loss of capping agents on the particle surface and the particle domain size from DLS, the graft density of the capping agents on the particles can be estimated assuming monolayer coverage on the spherical ceria particles. On the basis of this calculation, the graft density of capping agents is listed on the column fourth and seventh of Table 1 . The graft density of particles lies in the range of 0.94.1 molecules $/ \mathrm{nm}^{2}$ for in situ approach. However, the density for the agents varies in between 0.1 and 3.9 molecules $/ \mathrm{nm}^{2}$. This result suggests that in situ approach allows denser surface functionalization compared to the ex situ approach.

3.2. In Situ Polymerization of $\mathrm{CeO}_{2} / \mathrm{MMA}$ Dispersion. Both in situ and ex situ functionalized $\mathrm{CeO}_{2}$ particles were dispersed in MMA separately by keeping the weight fraction of particles at 0.03 with respect to MMA for each of particle/ MMA dispersion. The dispersions were subjected to bulk polymerization initiated by $\mathrm{BPO}$ at $60{ }^{\circ} \mathrm{C}$. The rate of polymerization, MW, and MWD were examined separately for neat MMA, MMA in the presence of capping agents only without the particles, MMA dispersion consisting of unfunctionalized, in situ functionalized, and ex situ functionalized ceria particles.

3.2.1. Conversion. At the initial stages of the experiments, the polymerization was carried out at $80{ }^{\circ} \mathrm{C}$. The decomposition of $\mathrm{BPO}$ at this temperature was fast $\left(t_{1 / 2}=\right.$ $1.4 \mathrm{~h}$ at $\left.85^{\circ} \mathrm{C}\right)^{30}$ such that the complete conversion is achieved in a short time period ( $30 \mathrm{~min}$, see Figure S3). The effect of particles on the polymerization lost its significance, and the temperature therefore is reduced to $60{ }^{\circ} \mathrm{C}$, where $t_{1 / 2}=100 \mathrm{~h}^{31}$ The conversion of MMA only (reference system) as a function of polymerization time is presented in panel a of Figure 3 with

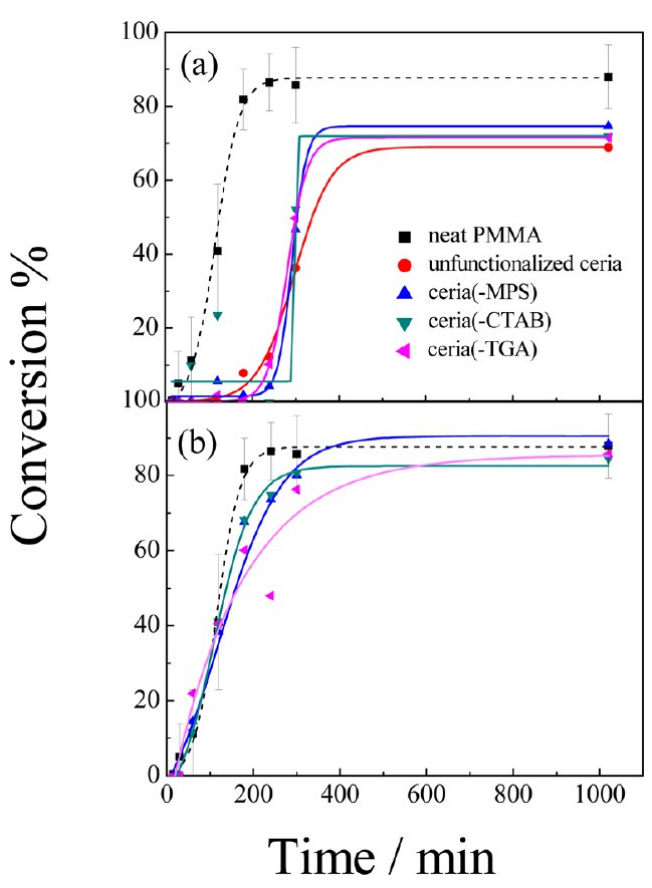

Figure 3. Conversion of MMA in the presence of (a) in situ and (b) ex situ functionalized $\mathrm{CeO}_{2}$ nanoparticles. The result of PMMA is shown in panels $\mathrm{a}$ and $\mathrm{b}$ as solid and dashed lines for comparison.

the dashed line. Not surprisingly, a sigmoidal increase with respect to time was obtained in the conversion curve most probably due to gel or Trommsdorf-Norrish effect. In free radical polymerization of vinyl monomers, the gel effect is commonly observed. Termination of a propagating radical is diffusion-controlled. As MMA is polymerized, the viscosity of the medium increases, and the medium is then saturated with the amount of propagating radicals. The rate of termination dramatically decreases. The exothermic nature of addition polymerization triggers the propagation step; hence, autoacceleration of polymerization inevitably takes place. However, in the presence of particles (both unfunctionalized- and functionalized- $\mathrm{CeO}_{2}$ ones), the autoacceleration appears at longer times. Particles of hard solid spheres in the polymerization medium may increase the viscosity of the polymerization mixture and hinder the diffusion of the radical chains so that polymerization is retarded by the particles regardless of the capping agents employed. Moreover, lower conversion was observed in nanocomposite formation compared to the reference system. This result suggests that in situ functionalized particles showed inhibition for the polymerization. However, the presence of ex situ functionalized ceria particles has no remarkable effect on the polymerization process (panel $b$ ). The reason of this result might be due to the loose and scarce grafting of the capping agents onto the particle surface.

3.2.2. Molecular Weight and Molecular Weight Distributions (MWD). Figure 4 demonstrates MWDs of PMMA synthesized in the presence of unfunctionalized and functionalized particles (both in situ and ex situ ones). For comparison, the same polymerization was carried out in the presence of neat capping agents only without the ceria particles. In addition, 


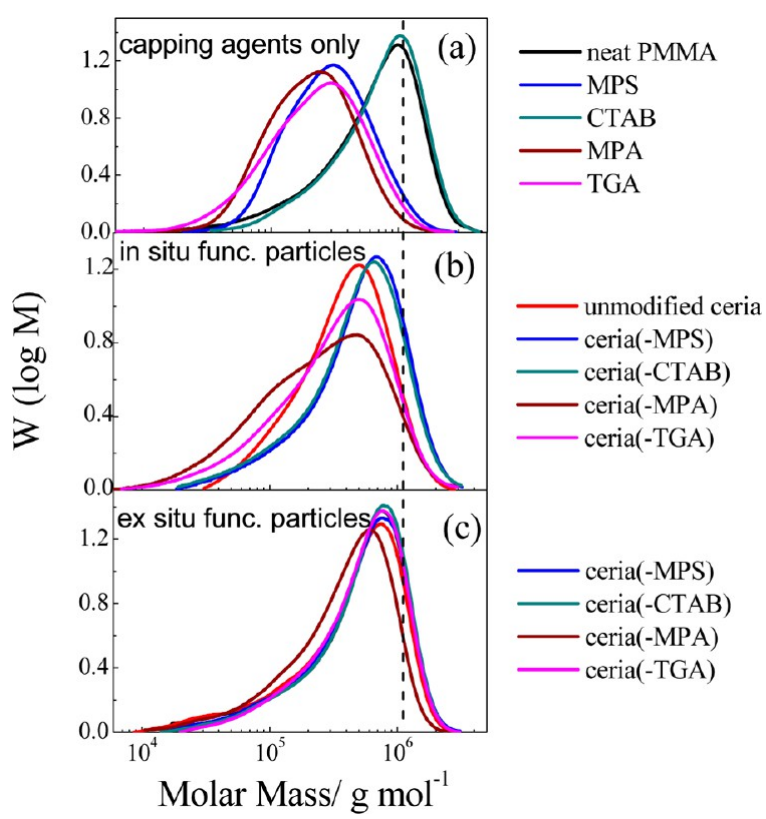

Figure 4. Molecular weight distribution of PMMA in presence of (a) cerium oxide functionalized in situ, (b) capping agents, and (c) ex situ over $1020 \mathrm{~min}$ of polymerization.

Table 2 gives number average molecular weight $\left(M_{\mathrm{n}}\right)$ and polydispersity index (PDI) of the MWDs given in Figure 4.

Capping Agents Only without the Ceria Particles. Panel a of Figure 4 shows MWDs of PMMA obtained in the presence of capping agents as well as reference PMMA (MMA only). PMMA obtained from the refence system has a wide bimodal distribution and the MW appears to be very high at $2.2 \times 10^{6}$ $\mathrm{g} \mathrm{mol}{ }^{-1}$. It is well-known that bulk polymerization is an uncontrolled way of polymerization such that chain population is not uniform and composed of both small and large chains. PMMA obtained in the presence of CTAB has a higher MW $\left(4.6 \times 10^{6} \mathrm{~g} \mathrm{~mol}^{-1}\right)$. Ghosh and Maity proposed a complexation taking place between $\mathrm{BPO}$ and $\mathrm{CTAB}$ in the free-radical polymerization of MMA. ${ }^{32}$ This complexation involves Coulombic interaction of BPO and cationic end of $\mathrm{CTAB}$ that causes bond breakage between oxygen atoms of peroxide (Figure S4). Then, the resulting cationic group behaves itself as a radical by leaving the complex. The colorless polymerization mixture turned into yellow upon this complexation. As a result, the efficiency of BPO was reduced so that higher MW chains are obtained. On the other hand, the PMMA chains obtained in the presence of thiol-containing agents appear to have lower molecular weight. The molecular weight of PMMA chains obtained in the presence of TGA, MPA, and
MPS was $1.4 \times 10^{6}, 1.5 \times 10^{6}$, and $2.1 \times 10^{6} \mathrm{~g} \mathrm{~mol}^{-1}$, respectively. It is well established that classical transfer agents reduce the molecular weight. Thiols have been recognized as chain transfer agents. The reactivity of a propagating radical is transferred to the sulfur atom. The sulfur radical is stable, and further propagation with a fresh monomer is not favorable. $^{31,25,30}$ Since small molecules diffuse faster in polymerization, the function of the thiol in such molecules appears to be more efficient. Thus, the smaller the molecule is, the lower the MW.

Unfunctionalized Particles. For PMMA composites prepared with unfunctionalized particles, $M_{n}$ was found to be $2.6 \times$ $10^{6} \mathrm{~g} \mathrm{~mol}^{-1}$. This $\mathrm{MW}$ is higher than that of the reference PMMA. Vibrational spectroscopy confirmed the interaction between $\mathrm{BPO}$ and ceria particle surface. Figure 5 presents the

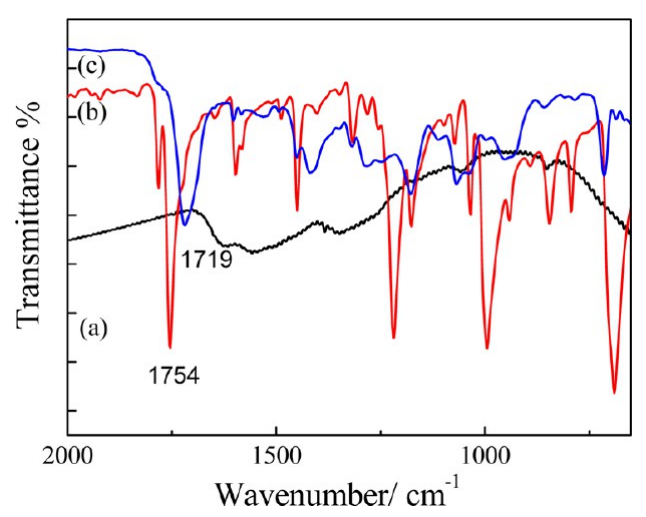

Figure 5. FTIR spectra of (a) unfunctionalized $\mathrm{CeO}_{2}$, (b) BPO, and (c) $\mathrm{CeO}_{2}-\mathrm{BPO}$ particles.

FTIR spectra of unfunctionalized particles, BPO itself, and unfunctionalized particles treated with $\mathrm{BPO}$ at $60{ }^{\circ} \mathrm{C}$. The unfunctionalized particles have weak and broad signals, i.e., do not have specific feature in this segment of the spectrum. On the other hand, BPO shows characteristic sharp vibrational signals. The fingerprint signals of BPO were found to be broadened and significantly shifted in BPO-treated ceria particles. For example, the stretching of the $\mathrm{C}=\mathrm{O}$ group of $\mathrm{BPO}$ is shifted from 1754 to $1719 \mathrm{~cm}^{-1}$ and full width halfmaximum of this signal is broadened $\sim 15 \mathrm{~cm}^{-1}$. The change in this spectral signal indicates the occurrence of a strong interaction of BPO with the ceria particle surface such that the efficiency of the initiator decreases. Thus, the chains synthesized in the presence of functionalized particles appear heavier than the reference PMMA.

In Situ Functionalized Particles. Panels b and $\mathrm{c}$ of Figure 4 show the MWDs of polymer obtained when in situ and ex situ

Table 2. Number Molecular Weight $\left(M_{n}\right)$ and Polydispersity Index (PDI) of PMMA in the Presence of Neat Capping Agents, and Cerium Oxide Functionalized In Situ and Ex Situ over 1020 min of Polymerization

\begin{tabular}{|c|c|c|c|c|c|c|c|c|}
\hline \multicolumn{3}{|c|}{ capping agent } & \multicolumn{3}{|c|}{ in situ } & \multicolumn{3}{|c|}{ ex situ } \\
\hline sample & $\begin{array}{c}M_{\mathrm{n}} / \mathrm{g} \mathrm{mol}^{-1} \\
\left(\times 10^{-5}\right)\end{array}$ & $\begin{array}{c}\text { PDI } \\
\left(M_{\mathrm{w}} / M_{\mathrm{n}}\right)\end{array}$ & sample & $\begin{array}{c}M_{\mathrm{n}} / \mathrm{g} \mathrm{mol}^{-1} \\
\left(\times 10^{-5}\right)\end{array}$ & $\begin{array}{c}\text { PDI } \\
\left(M_{\mathrm{w}} / M_{\mathrm{n}}\right)\end{array}$ & sample & $\begin{array}{c}M_{\mathrm{n}} / \mathrm{g} \mathrm{mol}^{-1} \\
\left(\times 10^{-5}\right)\end{array}$ & $\begin{array}{c}\text { PDI } \\
\left(M_{\mathrm{w}} / M_{\mathrm{n}}\right)\end{array}$ \\
\hline neat PMMA & 2.2 & 3.0 & & & & & & \\
\hline & & & unfunctionalized ceria & 2.6 & 1.9 & & & \\
\hline MPS & 2.1 & 1.7 & ceria(-MPS) & 2.9 & 2.2 & ceria(-MPS) & 2.8 & 2.3 \\
\hline СТАВ & 4.6 & 1.9 & ceria(-CTAB) & 2.7 & 2.3 & ceria(-CTAB) & 2.9 & 2.3 \\
\hline MPA & 1.5 & 1.8 & ceria(-MPA) & 1.3 & 3.0 & ceria(-MPA) & 2.1 & 2.3 \\
\hline TGA & 1.4 & 2.2 & ceria(-TGA) & 1.6 & 2.9 & ceria(-TGA) & 3.1 & 2.1 \\
\hline
\end{tabular}


funtionalized particles are used, respectively. A vertical line is shown passing through the mean of the distribution of PMMA (reference system). In the case of ceria(-CTAB), $M_{n}$ is $2.7 \times$ $10^{6} \mathrm{~g} \mathrm{~mol}^{-1}$. The chains are heavier than the reference PMMA and lighter than the chains obtained in the presence of free CTAB. This result suggests that CTAB is still active in the aforementioned complexation with BPO when it is immobilized to the particle surface; so that, higher molecular weight than the reference PMMA was obtained. On the other hand, its function remarkably decreases compared to the one obtained in the presence of free $\mathrm{CTAB}$ without ceria particles most probably due to steric hindrance. As a result, molecular weight appears to be lighter.

$$
\begin{aligned}
& \text { complexation } \quad \mathrm{BPO}+\mathrm{CTAB}(\text { surface }) \rightarrow \\
& \text { BPO: СТAB }(\text { surface })
\end{aligned}
$$

The use of thiol-capped particles yields PMMA chains that have a lower MW than that of the reference. The surface thiol groups are usual suspect for the decrease in MW. The propagating MMA radical is transferred to particle surface and further propagation is prevented.

$$
\begin{aligned}
& \text { chain transfer } \\
& \mathrm{R} \bullet+\mathrm{HS}(\text { surface }) \rightarrow \bullet \mathrm{S}(\text { surface })+\mathrm{RH}(\text { dead chain })
\end{aligned}
$$

However, the function of the thiol-capped particles in polymerization is varied and is somewhat different from their role when they are free in the monomer without particles. For example, although TGA was the most effective transfer agent used in this study due to its smaller size, ceria(-MPA) as a chain transfer agent functions more effectively than ceria(-TGA). Both MPA and TGA have a similar structure as well as similar grafting to the particle surface via carboxylic acid. In contrast to TGA, MPA has $\mathrm{CH}_{2}$ as its spacer group. When they are immobilized to the particle surface, ceria(-MPA) provides better mobility and flexibility for the thiol end-group and correspondingly better function in the transfer reaction. Another example for the variation of the role of the capping agents in polymerization when they are immobilized to the particle surface could be the role of ceria(-MPS). Surprisingly, it has almost no chain transfer function. Thiol is a good nucleophile, and it has tendency to attack silane groups in the molecule. In fact, the thiol group particularly becomes even more nucleophilic in a basic medium, which is the case for our particle synthesis where $\mathrm{NH}_{4} \mathrm{OH}$ is a precursor. MPS molecules on the particle surface undergo self-condensation between the silane and thiol groups ${ }^{33}$ and methanol is eliminated as a result of condensation. Thiol is, therefore, blocked by silane, and the function of MPS as a transfer agent is suppressed.

Ex Situ Functionalized Particles. $M_{\mathrm{n}}$ of PMMA obtained in the presence of ex situ functionalized particles was found in between 2.1 and $3.1 \times 10^{6} \mathrm{~g} \mathrm{~mol}^{-1}$. This range is relatively narrow compared to the chains produced in the presence of in situ functionalized particles $\left(1.3-2.9 \times 10^{6} \mathrm{~g} \mathrm{~mol}^{-1}\right)$. Therefore, the ex situ functionalized particles are not as effective as the in situ functionalized ones in the development of molecular weight. A similar result was also obtained for the conversion data (Figure 3). While in situ functionalized particles cause retardation and inhibition in conversion, the ex situ functionalized particles did not have a remarkable effect on conversion. Yet, FTIR revealed the adsorption of the capping agents on the particles, and TGA confirmed the presence of the capping agents on the particle surface. The reason for the inertness of the ex situ functionalized particle in the polymerization process may lie on the surface feature, i.e., the process of functionalization. While monolayer coverage of the capping agents can be achieved for the in situ functionalized particles, similar homogeneity cannot be obtained for the ex situ functionalized ones. In the process, the surface functionalization is carried out for the isolated and redispersed particles; therefore, the particles remain as aggregated and functionalization takes place for the large aggregated particle domains instead of functionalization of the single individual particles. Moreover, the capping agent molecules may not be distributed evenly throughout the surface of ex situ functionalized particles; rather, clusters of the capping agents may be adsorbed to the particles surface sparsely. Thus, the heterogeneous surface functionalization passivate neat ceria surface, however, does not contribute to the free radical polymerization.

3.3. Level of Nanoparticle Dispersion in PMMA. The dispersibility of particles in a polymer matrix is a key point in PNC. AFM was employed for the determination of the dispersibility of the particle domains. Table 3 shows the average

Table 3. Particle Domain Size in PMMA ${ }^{a}$

\begin{tabular}{cll} 
& \multicolumn{2}{c}{ particle domain size in PMMA } \\
\cline { 2 - 3 } Ceria particles used in the composites & in situ & ex situ \\
unfunctionalized ceria & $27 \pm 4$ & $25 \pm 9$ \\
ceria(-MPS) & $48 \pm 12$ & $61 \pm 10$ \\
ceria(-CTAB) & $21 \pm 6$ & $21 \pm 4$ \\
ceria(-TGA) & $21 \pm 3$ & $29 \pm 6$
\end{tabular}

${ }^{a}$ The measurement is based on the statistical treatment of particles domains in tapping mode AFM phase images.

diameter of particle domains measured by tapping mode AFM images. Figure 6 shows a representative set of the phase image
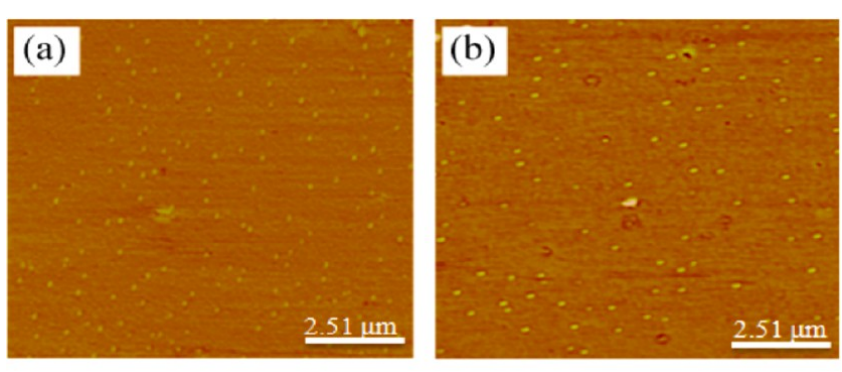

Figure 6. AFM images of in situ (a) and ex situ (b) TGA functionalized $\mathrm{CeO}_{2}$ nanoparticles.

of nanocomposites containing both in situ and ex situ functionalized ceria(-TGA) particles at 3 wt \%. In comparison to the particle size in MMA (see DLS results in Table 1), the domain size of both types of particles in PMMA appears to be smaller. Independent of the surface chemistry and approach employed in surface functionalization, the particles showed good dispersion in the polymer matrix. The domain size is $<50$ $\mathrm{nm}$, which is 1 order of magnitude smaller that their size in MMA. We think that the smaller domain size in the polymer might be the result of subsequent freeze-thaw processes we applied three times prior to in situ polymerization. Quenching the particle/MMA dispersion to very low temperature (to the temperature of liquid nitrogen) and subsequent heating to RT causes a serious volumetric change. Even if the nanosized 
particles contribute to the formation of aggregates/agglomerates, they eventually deagglomerated by the end of this freeze and thaw process.

3.4. Thermal Properties of the Nanocomposites. Figure S5 shows degradation profiles of the PMMA composites in air. Based on these profiles, the derivative of thermal degradation (DTG) of both neat PMMA and PMMA/CeO composites prepared by in situ functionalized nanoparticles was obtained (Figure 7). Three discrete degradation steps were

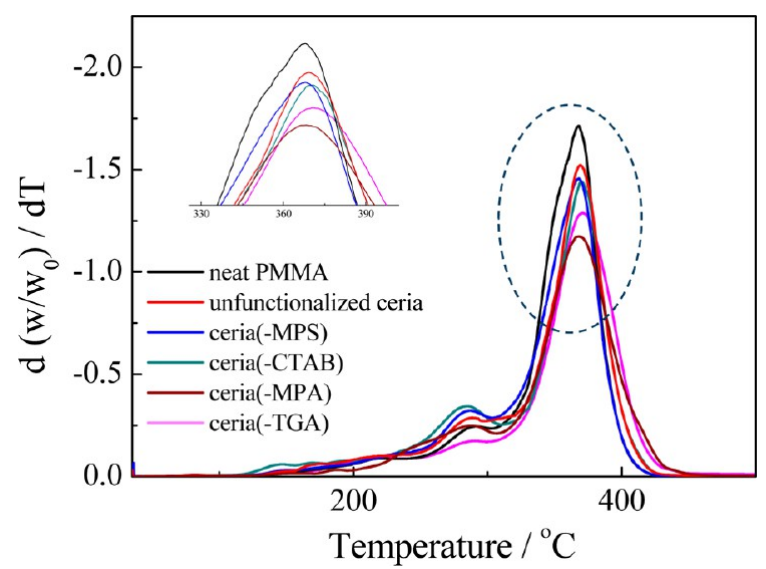

Figure 7. DTG curves of thermal decomposition of neat PMMA and its composites prepared by both unfunctionalized and functionalized $\mathrm{CeO}_{2}$ nanoparticles.

clearly observed in the thermogram at around 160, 280, and $370{ }^{\circ} \mathrm{C}$. This result agrees perfectly with the literature of the decomposition of PMMA. ${ }^{34,35}$ It is well established that the mechanism of radical termination in polymerization process has strong influence on the thermal properties of the resulting composite material. There are two termination mechanisms: (i) combination and (ii) disproportionation. ${ }^{30}$ Both reactions yield abnormal linkages. The former one gives head-to-head linkages; in fact head-to-tail is the normal type of bonding for monomeric units. The latter one presents vinylidene end chains that unzip the chain under degradation conditions. These first two mass losses correspond to the decomposition of the head-to-head linkage and the unzipping of the vinylidene chain end, respectively. The main mass loss occurs around 370 ${ }^{\circ} \mathrm{C}$ due to the random chain scission. The intensity of this signal decreases for the chains obtained in the presence of ceria nanoparticles (both unfunctionalized and functionalized ones) and may be correlated with the molecular weight of the in situ formed PMMA chains. For example, when thiol capped ceria particles are used, the intensity of the signal decreases. The degradation of lower molecular weight chains readily occurs so that the intensity of the mass loss at $370{ }^{\circ} \mathrm{C}$ increases as the molecular weight decreases.

Interfacial attraction between particles and the surrounding PMMA matrix has significant influence on many macroscopic properties for instance glass transition temperature $\left(T_{\mathrm{g}}\right)$ of the polymer composites. The presence of the nanoparticles might demonstrate either attractive or repulsive interaction; thus, a change in $T_{\mathrm{g}}$ is an evidence of these interactions. DSC can provide information about the comparative interaction of the particles functionalized either in situ or ex situ with the surrounding polymer matrix. When this interaction is attractive, polymer chains those are directly in contact with nanoparticles are decelerated and an increase in $T_{\mathrm{g}}$ occurs. ${ }^{36}$ DSC curve and the value of each composite were given in Figure S6 and Table 4 , respectively. The effects of neat surfactants on $T_{\mathrm{g}}$ of the

Table 4. Glass Transition Temperatures $\left(T_{\mathrm{g}} /{ }^{\circ} \mathrm{C}\right)$ of PMMA Prepared in the Presence of Capping Agents, Ceria Nanoparticles Surface Functionalized Both In Situ and Ex Situ Approaches ${ }^{a}$

$\begin{array}{lcccc} & \begin{array}{c}\text { PMMA } \\ \text { with } \\ \text { capping }\end{array} & \text { sample } & \begin{array}{c}\text { PMMA with in } \\ \text { situ } \\ \text { functionalized } \\ \text { ceria }\end{array} & \begin{array}{c}\text { PMMA with ex } \\ \text { situ } \\ \text { functionalized } \\ \text { ceria }\end{array} \\ \text { MPS } & 103 & \text { ceria(-MPS) } & 123 & 111 \\ \text { CTAB } & 115 & \text { ceria(-CTAB) } & 114 & 117 \\ \text { MPA } & 98 & \text { ceria(-MPA) } & 117 & 116 \\ \text { TGA } & 96 & \text { ceria(-TGA) } & 123 & 113\end{array}$

${ }^{a}$ Note that $T_{\mathrm{g}}$ of the PMMA composite prepared by unfunctionalized ceria particles is $116^{\circ} \mathrm{C}$.

polymer were given in the second column of Table 4 . While $T_{\mathrm{g}}$ of neat PMMA was $108{ }^{\circ} \mathrm{C}$, this temperature decreased to 103 , 98 , and $96{ }^{\circ} \mathrm{C}$ in the presence of capping agents MPS, MPA, and TGA. The addition of free capping agents without grafting to ceria nanoparticles decreases the $T_{\mathrm{g}}$ value. The capping agents are smaller molecules compared to PMMA chains and they act as a plasticizer that may reduce $T_{\mathrm{g}}$. The glass transition temperature is increased to $116^{\circ} \mathrm{C}$ when PMMA is prepared in the presence of unfunctionalized particles most probably due to the interaction between surface hydroxyls and the acrylate group of the PMMA chains. Similarly, a remarkable enhancement in the $T_{\mathrm{g}}$ value was observed by the addition of particles regardless of the surface feature (column four and five of Table 4). An increase in $T_{\mathrm{g}}$ can be the consequence of interactions between free functional - $\mathrm{SH}$ groups of the capping agents and carbonyl group of PMMA chains. In contrast, there is no any remarkable change between $T_{\mathrm{g}}$ of the polymer chains synthesized by the presence of neat CTAB and CTAB functionalized $\mathrm{CeO}_{2}$ nanoparticles. The plasticizing effect of aliphatic chains-C18 may offset the increase of $T_{\mathrm{g}}$ due to the presence of nanoparticles. The composites prepared by ex situ functionalized particles do not show strong variation in $T_{\mathrm{g}}$ and lie between 111 and $117^{\circ} \mathrm{C}$, considering the $T_{\mathrm{g}}$ of composites prepared by unfunctionalized particles is $116^{\circ} \mathrm{C}$. One can conclude that the interaction of the ex situ functionalized particles and surrounding PMMA chains is lower compared to the interaction with in situ functionalized ones with the PMMA matrix.

\section{CONCLUSION}

PMMA composites including ceria nanoparticles were prepared by free radical in situ bulk polymerization using BPO as initiator. Surface functionalization of the $\mathrm{CeO}_{2}$ nanoparticles was carried out using various surfactant molecules having different chemistry (TGA, MPA, MPS, and CTAB) employing both in situ and ex situ approaches. We have shown that how the particle surface functionalization is performed plays a decisive role in the polymerization process. While in situ functionalized $\mathrm{CeO}_{2}$ nanoparticles contributed to polymerization, the influences of ex situ functionalized ones were not noteworthy although the same surfactant molecules were used with the same amount. For example, in situ functionalized particles cause retardation and inhibition in the polymerization; however, a remarkable influence was not observed when ex situ functionalized particles were employed. The result is 
presumably due to the fact that the in situ approach provides densely and homogeneously covered the particle surface. The freeze and thaw process was found to be useful in the process of in situ polymerization. It causes severe deagglomeration, even for the unfunctionalized particles that strongly contribute to aggregation/agglomeration in MMA. A systematic study focusing on the effect of the substrate (silica, zincite, and titania), level of particle loading, polymerization temperature, and type of free-radical initiator may shed light on the detailed mechanism of the process of in situ polymerization.

\section{ASSOCIATED CONTENT}

\section{S Supporting Information}

XRD pattern of the particles, FTIR of the particle, conversion of MMA at $80^{\circ} \mathrm{C}$, scheme of complexation between BPO and CTAB, TGA of PMMA composites, and DSC of the PMMA composites. This material is available free of charge via the Internet at http://pubs.acs.org.

\section{AUTHOR INFORMATION}

\section{Corresponding Author}

*E-mail: mdemir@iyte.edu.tr. Tel.: +90 23275075 11. Fax: +902327507509.

Notes

The authors declare no competing financial interest.

\section{ACKNOWLEDGMENTS}

M.M.D. thanks The Scientific and Technological Research Council of Turkey (TUBITAK) for the research project encoded with TBAG-109T905. The authors thank Dr. Rafael Muñoz-Espi for GPC analysis and TEM imaging and Center for Materials Research of IZTECH for the rest of the measurements.

\section{REFERENCES}

(1) Lin, Z.; Cheng, Y. R.; Lu, H.; Zhang, L. A.; Yang, B. Preparation and Characterization of Novel Zns/Sulfur-Containing Polymer Nanocomposite Optical Materials with High Refractive Index and High Nanophase Contents. Polymer 2010, 51, 5424.

(2) Demir, M. M.; Castignolles, P.; Akbey, U.; Wegner, G. In Situ Bulk Polymerization of Dilute Particle/Mma Dispersions. Macromolecules 2007, 40, 4190.

(3) Tjong, S. C. Structural and Mechanical Properties of Polymer Nanocomposites. Mater. Sci. Eng. R-Rep. 2006, 53, 73.

(4) LeBaron, P. C.; Wang, Z.; Pinnavaia, T. J. Polymer-Layered Silicate Nanocomposites: An Overview. Appl. Clay Sci. 1999, 15, 11.

(5) Moniruzzaman, M.; Winey, K. I. Polymer Nanocomposites Containing Carbon Nanotubes. Macromolecules 2006, 39, 5194.

(6) Gorrasi, G.; Tortora, M.; Vittoria, V.; Pollet, E.; Lepoittevin, B.; Alexandre, M.; Dubois, P. Vapor Barrier Properties of Polycaprolactone Montmorillonite Nanocomposites: Effect of Clay Dispersion. Polymer 2003, 44, 2271.

(7) Nazarenko, S.; Meneghetti, P.; Julmon, P.; Olson, B. G.; Qutubuddin, S. Gas Barrier of Polystyrene Montmorillonite Clay Nanocomposites: Effect of Mineral Layer Aggregation. J. Polym. Sci. Pt. B-Polym. Phys. 2007, 45, 1733.

(8) Ahmadi, S. J.; Huang, Y. D.; Li, W. Synthetic Routes, Properties and Future Applications of Polymer-Layered Silicate Nanocomposites. J. Mater. Sci. 2004, 39, 1919.

(9) Olad, A.; Rasouli, H. Enhanced Corrosion Protective Coating Based on Conducting Polyaniline/Zinc Nanocomposite. J. Appl. Polym. Sci. 2010, 115, 2221.

(10) Wang, C.; Dong, B.; Gao, G. Y.; Xu, M. W.; Li, H. L. A Study on Microhardness and Tribological Behavior of Carbon Nanotubes
Reinforced Amma-Cnts Copolymer Nanocomposites. Mater. Sci. Eng. A 2008, 478, 314.

(11) Demir, M. M.; Wegner, G. Challenges in the Preparation of Optical Polymer Composites with Nanosized Pigment Particles: A Review on Recent Efforts. Macromol. Mater. Eng. 2012, 297, 838.

(12) Anzlovar, A.; Orel, Z. C.; Zigon, M. Poly(Methyl Methacrylate) Composites Prepared by in Situ Polymerization Using Organophillic Nano-to-Submicrometer Zinc Oxide Particles. Eur. Polym. J. 2010, 46, 1216.

(13) Zhang, L.; Li, F.; Chen, Y. W.; Wang, X. F. Synthesis of Transparent Zno/Pmma Nanocomposite Films through Free-Radical Copolymerization of Asymmetric Zinc Methacrylate Acetate and in Situ Thermal Decomposition. J. Lumin. 2011, 131, 1701.

(14) Wu, K. L.; Chou, S. C.; Cheng, Y. Y. Comparison of Polyimide/ Multiwalled Carbon Nanotube (Mwnt) Nanocomposites by in Situ Polymerization and Blending. J. Appl. Polym. Sci. 2010, 116, 3111.

(15) Zhou, C. J.; Qiu, X. Y.; Zhuang, Q. X.; Han, Z. W.; Wu, Q. L. In Situ Polymerization and Photophysical Properties of Poly(P-Phenylene Benzobisoxazole)/Multiwalled Carbon Nanotubes Composites. J. Appl. Polym. Sci. 2012, 124, 4740.

(16) Baniasadi, H.; Ramazani, A.; Nikkhah, S. J. Investigation of in Situ Prepared Polypropylene/Clay Nanocomposites Properties and Comparing to Melt Blending Method. Mater. Des. 2010, 31, 76.

(17) Demir, M. M.; Memesa, M.; Castignolles, P.; Wegner, G. Pmma/Zinc Oxide Nanocomposites Prepared by in Situ Bulk Polymerization. Macromol. Rapid Commun. 2006, 27, 763.

(18) Hess, S.; Demir, M. M.; Yakutkin, V.; Baluschev, S.; Wegner, G. Investigation of Oxygen Permeation through Composites of Pmma and Surface-Modified Zno Nanoparticles. Macromol. Rapid Commun. 2009, 30, 394.

(19) Campos, C. L.; Roldan, C.; Aponte, M.; Ishikawa, Y.; Cabrera, C. R. Preparation and Methanol Oxidation Catalysis of Pt-Ceo2 Electrode. J. Electroanal. Chem. 2005, 581, 206.

(20) Parlak, O.; Demir, M. M. Toward Transparent Nanocomposites Based on Polystyrene Matrix and Pmma-Grafted Ceo2 Nanoparticles. ACS Appl. Mater. Interfaces 2011, 3, 4306.

(21) Corma, A.; Atienzar, P.; Garcia, H.; Chane-Ching, J. Y. Hierarchically Mesostructured Doped Ceo2 with Potential for SolarCell Use. Nat. Mater. 2004, 3, 394.

(22) Lawrence, N. J.; Brewer, J. R.; Wang, L.; Wu, T. S.; WellsKingsbury, J.; Ihrig, M. M.; Wang, G. H.; Soo, Y. L.; Mei, W. N.; Cheung, C. L. Defect Engineering in Cubic Cerium Oxide Nanostructures for Catalytic Oxidation. Nano Lett. 2011, 11, 2666.

(23) Lee, T. Y.; Bowman, C. N. The Effect of Functionalized Nanoparticles on Thiol-Ene Polymerization Kinetics. Polymer 2006, 47, 6057.

(24) Stevens, M. P. Polymer Chemistry: An Introduction; Oxford University Press, Inc.: New York, 1999.

(25) Murray, E. P.; Tsai, T.; Barnett, S. A. A Direct-Methane Fuel Cell with a Ceria-Based Anode. Nature 1999, 400, 649.

(26) Saric, M.; Dietsch, H.; Schurtenberger, P. In Situ Polymerisation as a Route Towards Transparent Nanocomposites: Time-Resolved Light and Neutron Scattering Experiments. Colloid Surf. A 2006, 291, 110

(27) Tunusoglu, O.; Munoz-Espi, R.; Akbey, U.; Demir, M. M. Surfactant-Assisted Formation of Organophilic Ceo2 Nanoparticles. Colloid Surf. A 2012, 395, 10.

(28) Taguchi, M.; Takami, S.; Naka, T.; Adschiri, T. Growth Mechanism and Surface Chemical Characteristics of Dicarboxylic Acid-Modified Ceo2 Nanocrystals Produced in Supercritical Water: Tailor-Made Water-Soluble Ceo2 Nanocrystals. Cryst. Growth Des. 2009, 9, 5297.

(29) Kepenekci, O.; Emirdag-Eanes, M.; Demir, M. M. Effect of Alkali Metal Hydroxides on the Morphological Development and Optical Properties of Ceria Nanocubes under Hydrothermal Conditions. J. Nanosci. Nanotechnol. 2011, 11, 3565.

(30) Odian, G. Principles of Polymerization, 4th ed.; John Wiley \& Sons, Inc.: New York, 2004. 
(31) Bartlett, P. D.; Hiatt, R. R. A Series of Tertiary Butyl Peresters Showing Concerted Decomposition. J. Am. Chem. Soc. 1958, 80, 1398. (32) Ghosh, P.; Maity, S. N. Polymerization of Methyl-Methacrylate with Use of Acetyl Trimethyl Ammonium Bromide Benzoyl Peroxide Combination as Initiating System. Eur. Polym. J. 1978, 14, 855.

(33) Jacobine, A. F. Radiation Curing in Polymer Science and Technology; Elsevier: London, 1993.

(34) Demir, M. M.; Koynov, K.; Akbey, U.; Bubeck, C.; Park, I.; Lieberwirth, I.; Wegner, G. Optical Properties of Composites of Pmma and Surface-Modified Zincite Nanoparticles. Macromolecules 2007, 40, 1089.

(35) Kashiwagi, T.; Morgan, A. B.; Antonucci, J. M.; VanLandingham, M. R.; Harris, R. H.; Awad, W. H.; Shields, J. R. Thermal and Flammability Properties of a Silica-Poly(Methylmethacrylate) Nanocomposite. J. Appl. Polym. Sci. 2003, 89, 2072.

(36) Jancar, J.; Douglas, J. F.; Starr, F. W.; Kumar, S. K.; Cassagnau, P.; Lesser, A. J.; Sternstein, S. S.; Buehler, M. J. Current Issues in Research on Structure-Property Relationships in Polymer Nanocomposites. Polymer 2010, 51, 3321. 